\title{
Pan-cancer systematic identification of IncRNAs associated with cancer prognosis
}

\author{
Matthew Ung ${ }^{1}$, Evelien Schaafsma ${ }^{1}$, Daniel Mattox ${ }^{2}$, George L Wang ${ }^{1}$, Chao Cheng ${ }^{\text {Corresp. } 1,3,4,5}$ \\ ${ }^{1}$ Department of Molecular and Systems Biology, Dartmouth College, Hanover, New Hampshire, United States \\ 2 Department of Computer Science, Dartmouth College, Hanover, New Hampshire, United States \\ 3 Department of Medicine, Baylor College of Medicine, Houston, TX, United States \\ 4 The Institute for Clinical and Translational Research, Baylor College of Medicine, Houston, TX, United States \\ 5 Department of Biomedical Data Science, Geisel School of Medicine at Dartmouth, Lebanon, New Hampshire, United States \\ Corresponding Author: Chao Cheng \\ Email address: chao.cheng@dartmouth.edu
}

Background. The "dark matter" of the genome harbors several non-coding RNA species including IncRNAs, which have been implicated in neoplasias but remain understudied. RNA-seq has provided deep insights into the nature of IncRNAs in cancer but current RNAseq data are rarely accompanied by longitudinal patient survival information. In contrast, a plethora of microarray studies have collected these clinical metadata that can be leveraged to identify novel associations between gene expression and clinical phenotypes. Methods. In this study, we developed an analysis framework that computationally integrates RNA-seq and microarray data to systematically screen 9,463 IncRNAs for association with mortality risk across 20 cancer types. Results. In total, we identified a comprehensive list of associations between IncRNAs and patient survival and demonstrate that these prognostic IncRNAs are under selective pressure and may be functional. Our results provide valuable insights that facilitate further exploration of IncRNAs and their potential as cancer biomarkers and drug targets. 
1 Pan-cancer systematic identification of IncRNAs associated with cancer

2 prognosis

3 Running title: Pan-cancer prognostications of IncRNAs

4

5 Matthew H. Ung ${ }^{1}$, Evelien Schaafsma1 ${ }^{1}$, Daniel E. Mattox ${ }^{2}$, George L. Wang ${ }^{1}$, Chao

6 Cheng ${ }^{1,3,4,5 *}$

7

$8{ }^{1}$ Department of Molecular and Systems Biology, Dartmouth College, Hanover, New

9 Hampshire, United States; ${ }^{2}$ Department of Computer Science, Dartmouth College,

10 Hanover, New Hampshire, United States; ${ }^{3}$ Department of Biomedical Data Science,

11 Geisel School of Medicine at Dartmouth, Lebanon, New Hampshire, United States; 4

12 Department of Medicine, Baylor College of Medicine, Houston, Texas, United States;

13 and ${ }^{5}$ The Institute for Clinical and Translational Research, Baylor College of Medicine,

14 Houston Texas, United States.

15

*Corresponding author: Chao Cheng

17 Address: One Baylor Plaza, Houston, TX 77030, USA.

18 Email: chao.cheng@bcm.edu, phone: (713) 7983332

19

20 Keywords: IncRNA, prognosis, microarray, RNA-seq, TCGA

21 Figure count: 6 


\section{Abstract}

23 Background. The "dark matter" of the genome harbors several non-coding RNA 24 species including IncRNAs, which have been implicated in neoplasias but remain 25 understudied. RNA-seq has provided deep insights into the nature of IncRNAs in cancer 26 but current RNA-seq data are rarely accompanied by longitudinal patient survival 27 information. In contrast, a plethora of microarray studies have collected these clinical 28 metadata that can be leveraged to identify novel associations between gene expression 29 and clinical phenotypes.

30 Methods. In this study, we developed an analysis framework that computationally

31 integrates RNA-seq and microarray data to systematically screen 9,463 IncRNAs for 32 association with mortality risk across 20 cancer types.

33 Results. In total, we identified a comprehensive list of associations between IncRNAs

34 and patient survival and demonstrate that these prognostic IncRNAs are under selective 35 pressure and may be functional. Our results provide valuable insights that facilitate 36 further exploration of IncRNAs and their potential as cancer biomarkers and drug 37 targets. 
38

39

40

41

42

43

44

45

46

47

48

49

50

51

52

53

54

55

56

57

58

59

60

\section{Introduction}

Long non-coding RNAs (IncRNAs) constitute a relatively unexplored repertoire of gene products that exhibit diverse functions and are involved in several biological processes. As such, the ENCODE consortium reported that $80 \%$ of the genome is transcribed into a variety of functional products including non-coding RNAs (Consortium, 2012). Several high-level characteristics of IncRNAs provide evidence that they are indeed functional, including their association with chromatin signatures of active transcription, being transcribed by RNA polymerase II, and undergoing post-transcriptional modifications such as polyadenylation and alternative splicing (Wang \& Chang, 2011; Rinn \& Chang, 2012; Kung, Colognori \& Lee, 2013). The mechanisms by which IncRNAs regulate biological processes have not been studied in detail but evidence suggest that they can function at the transcriptional, post-transcriptional, and post-translational level by acting as biological signals, decoys, guides, and scaffolds (Wang \& Chang, 2011; Rinn \& Chang, 2012; Kung, Colognori \& Lee, 2013). Moreover, the organization of IncRNAs across the genome is quite diverse in that they can be transcribed from intergenic regions, sites anti-sense to protein coding genes, bi-directional promoters, or within gene introns (Ponting, Oliver \& Reik, 2009; Kung, Colognori \& Lee, 2013).

Having been previously referred to as transcriptomic noise or "junk" DNA, IncRNAs are now being investigated as molecular players in several disease processes including cancer (Mattick \& Makunin, 2006; Esteller, 2011; Consortium, 2012; Sahu, Singhal \& Chinnaiyan, 2015; Schmitt \& Chang, 2016; Bartonicek, Maag \& Dinger, 2016; Evans, Feng \& Chinnaiyan, 2016). In this particular context, IncRNAs have been implicated in all hallmarks of cancer including sustaining proliferative signaling, evading 
61 growth suppressors, enabling replicative immortality, activating invasion and metastasis,

62 inducing angiogenesis, and resisting cell death (Hanahan \& Weinberg, 2000; Gutschner

63 \& Diederichs, 2012; Ali et al., 2018; Chiu et al., 2018). Aberrant expression of IncRNAs

64 might be due to their close association with certain key driver genes (Ashouri et al.,

65 2016) or the establishment of cancer-specific genomic features in IncRNA loci itself,

66 including mutational events, methylation, copy number, and SNP events (lyer et al.,

67 2015; Yan et al., 2015). Several studies have performed pan-cancer screens for

68 IncRNAs involved in the disease and found that several of them were differentially

69 expressed compared to normal samples, revealing their potential as biomarker

70 candidates (Cabanski et al., 2015; Yan et al., 2015; Byron et al., 2016; Ching et al.,

71 2016). For instance, PCA3 is a IncRNA that is currently approved for clinical use as a

72 prostate cancer diagnostic biomarker and can be detected in patient urine samples

73 (Schalken, J.A., 2002). Thus, dissecting the molecular characteristics of these

74 understudied RNAs and their associations with disease phenotypes may yield findings

75 that can be translated into the clinic.

76 In light of these findings, there is a paucity of patient samples with matched RNA-

77 seq data and clinical information which limits the ability to perform pan-cancer screening

78 for prognostic IncRNAs. Furthermore, few of these matched datasets contain sufficiently

79 long follow-up times which limits statistical power when performing survival analyses,

80 especially in cancer types where patients exhibit high survival rates (Clark et al., 2003).

81 In stark contrast, there is a plethora of microarray gene expression data that are

82 available, many of which are accompanied by comprehensive clinical information with

83 long follow-up times. 
Thus, using primarily protein-coding gene expression from microarray to infer the

expression of their non-coding counterparts can re-purpose these valuable data and generate novel hypotheses about IncRNAs associated with patient mortality across several cancer types. To this end, multiple studies have attempted to utilize data from microarray to make inferences about IncRNA activity and their clinical relevance. Du et al. re-annotated probes from microarray data to identify prognostic transcriptional activity for $\sim 10,000$ IncRNAs in prostate cancer, glioblastoma, ovarian cancer, and lung squamous cell carcinoma (Du et al., 2013). From this screen, they identified novel IncRNA markers of mortality risk and validated several of them experimentally. Furthermore, Guo et al. performed a "guilt-by-association" analysis whereby IncRNAs that share an edge with prognostic protein coding genes in a biological network defined a priori were also considered prognostic (Guo, Yao \& Jiang, 2016). Although these studies have provided valuable insights into IncRNA biology, the reannotation of microarray probes might have missed prognostic IncRNAs not captured by microarray probes. In addition, IncRNA inference based on known protein coding target genes might bias IncRNA expression if not all target genes are known.

Therefore, we introduce a IncRNA inference approach that generates cancerspecific weighted IncRNA regulon network profiles de novo using RNA-seq data from TCGA (The Cancer Genome Atlas), and apply them to infer IncRNA expression in the PRECOG (Gentles et al., 2015) and METABRIC (Curtis et al., 2012) microarray compendia, which provide expression of protein-coding genes but not for most IncRNAs. Afterwards, we systematically interrogated each IncRNA to identify those that significantly associate with patient prognosis using clinical metadata included in the 
107 microarray studies. In total we screened 9,463 unique IncRNAs across 20 different

108 cancer types to identify novel associations.

109

110 Materials \& Methods

111 Data source and pre-processing

112 The IncRNA gene list with Ensembl IDs was derived from the TANRIC resource (Li et

113 al., 2015). Level 3 RNA-seq count data from tumor samples encompassing 23 different

114 cancer types along with corresponding clinical information were downloaded from the

115 National Cancer Institute's Genomic Data Commons data portal

116 (https://portal.gdc.cancer.gov/). The count data was normalized by library size and

117 subjected to a variance stabilizing transformation implemented using DESeq2 (Love,

118 Huber \& Anders, 2014). This transforms the expression values so that they are

119 homoskedastic by fitting the dispersion to a negative binomial distribution. 141

120 microarray gene expression datasets across 20 cancer types were downloaded from

121 the PRECOG resource (Gentles et al., 2015). Normalized breast cancer gene

122 expression and copy number alteration datasets from METABRIC $(n=1992)$ were

123 downloaded from the European Genome-Phenome Archive (http://www.ebi.ac.uk/ega/)

124 under the accession number EGAS00000000083. CRISPRi screening data on

125 functional IncRNAs in MDA-MB-231 and K562 cell lines were downloaded from Liu et al.

126 (Liu et al., 2017).

127

128 Construction of cancer-specific regulons 
129 The ARACNe-AP algorithm was applied to each processed TCGA RNA-seq cancer

130 dataset using the TANRIC IncRNA Ensembl gene IDs as the regulator mapping set.

131 Briefly, ARACNe-AP calculates the mutual information between a IncRNA and potential

132 target genes and removes edges that are unlikely to represent a biological link using the

133 concept of data processing inequality (Margolin et al., 2006; Lachmann et al., 2016). We

134 implemented the algorithm using 100 bootstrap iterations and a p-value threshold of

135 0.01. Each regulon in the cancer-specific network consisted of a IncRNA and its

136 associated genes. Each edge in the regulon was assigned a weight using the mutual

137 information scores outputted by ARACNe-AP (Alvarez et al., 2016). The mutual

138 information scores were divided by the maximum score within each regulon so that they

139 had a range from 0 to 1 . The sign of the edge was assigned by computing a Pearson

140 correlation coefficient between the IncRNA's expression and the associated gene's

141 expression across the samples. Since genes in a regulon are positively or negatively

142 correlated with the corresponding IncRNA in a specific cancer type, their expression can

143 be used to impute the expression level of the IncRNA.

Inference of IncRNA expression in microarray datasets

146 For each cancer-specific regulon, we defined a pair of profiles--the genes with a positive

147 weight were assigned to an "up-regulated" profile and the genes with a negative weight

148 were assigned to a "down-regulated" profile. In the up-regulated profile, all genes that

149 had a negative weight were assigned a value of 0 and all genes in the down-regulated

150 profile that had a positive weight were assigned a value of 0 . The values in the down-

151 regulated profile were then forced to be positive. Only profiles with 20 or more 
152 associated genes were used. Thus, each IncRNA was assigned two regulon weight

153 profiles that captures the magnitude and direction of the genes it was associated with.

154 Genes with higher weights in the two profiles will contribute more to the imputation of

155 IncRNA expression.

156

157 After constructing the regulon weight profiles, IncRNA expression was inferred in 158 microarray datasets by using the regulon weight profile derived from the same cancer

159 type (or most related cancer type) as the microarray experiment (Supplementary

160 Results). To apply the regulon weight profiles to infer IncRNA expression in microarray

161 samples, we utilized the BASE algorithm (Cheng et al., 2007) which outputs a predicted

162 expression value for each IncRNA in every patient sample. BASE imputes the relative

163 expression level of a IncRNA based on the expression of genes that it correlates with

164 (i.e. regulon genes). Specifically, the algorithm sorts each patient's gene expression 165 profile from highest to lowest expressed genes and weights them using the two regulon weight profiles. BASE then calculates a running sum statistic by moving down the profile

167 and calculating a foreground function which captures the weighted enrichment of the IncRNA's associated genes at the top and bottom of the patient's gene expression profile. The foreground function is then compared to a background function and the maximum deviation between the foreground and background functions is computed.

171 The maximum deviation calculated from the down-regulated profile is subtracted from

172 the maximum deviation calculated from the up-regulated profile to yield a pre-inferred

173 IncRNA expression value (pre-iExpr). For a IncRNA, if positively associated genes tend

174 to be highly expressed (at the top of the expression profile) in a tumor sample while 
175 negatively associated genes tend to be lowly expressed (at the bottom of the

176 expression profile), we will observe a high pre-iExpr value. The patient's gene

177 expression profile is then randomly permuted and the procedure is repeated; this is

178 performed 1000 times to yield a null pre-iExpr distribution. The pre-iExpr score is then

179 normalized by dividing by the mean of the null pre-iExpr values to yield the final inferred 180 expression of the IncRNA (iExpr). The formulas describing the details of this algorithm is 181 provided in the Supplementary Methods.

183 Systematic inference of prognostic IncRNAs

184 A univariate Cox proportional hazards model was fit to the inferred and actual

185 expression values for each IncRNA, separately for TCGA, PRECOG, and METABRIC 186 datasets. Actual expression was available for a small set of IncRNAs in the PRECOG 187 and METABRIC datasets and were used for downstream validation. From the models, 188 z-scores were calculated by dividing the Cox regression coefficient by its standard error.

189 A z-score $<0$ indicates that a IncRNA is protective and positively associated with

190 survival. Conversely, a z-score $>0$ indicates that a IncRNA is hazardous and negatively

191 associated with survival.

192 In the PRECOG dataset, there were several microarray datasets belonging to the 193 same cancer type. After computing the inferred expression for all IncRNAs within each

194 dataset, we fitted a univariate Cox regression model to measure the association

195 between a IncRNA and all-cause or disease-specific mortality (if available). z-scores

196 were extracted from the fitted models and a meta z-score was calculated for each

197 IncRNA across all the microarray datasets belonging to the same cancer type. The meta 
z-score was calculated using weighted Stouffer's z-score method using the dataset

199 sample size as weights. A meta z-score $<1$ indicates a positive association and a meta

200 z-score >1 indicates a negative association with survival. In addition, robust meta z-

201 scores were calculated for each IncRNA by leaving out the dataset yielding the most

202 significant association and repeating the procedure. Meta p-values were calculated from

203 the meta z-scores by referring to the standard normal distribution. Meta p-values were

204 adjusted for each cancer type using Benjamini-Hochberg and Bonferroni multiple testing

205 correction methods. Kaplan-Meier analysis of IncRNAs was performed by dichotomizing

206 patients into high $(>0)$ and low $(<0)$ inferred IncRNA expression groups and performing

207 a log-rank test to calculate statistical significance.

208 In the METABRIC dataset, a multiple Cox regression model was applied and

209 included age at diagnosis, tumor size, stage, ER, and HER2 status as covariates.

210 Disease-specific mortality was used as the outcome.

211

212 Validation of survival analysis

213 To compare survival results across datasets, we performed two validation analyses: 1.

214 Cross-dataset analysis comparing Cox regression results using actual IncRNA

215 expression from TCGA with results using inferred IncRNA expression from PRECOG,

216 and 2. Within-dataset comparison of survival results generated by models fitted to

217 inferred or actual IncRNA expression in PRECOG and METABRIC. Pearson correlation

218 was used to evaluate the consistency between IncRNA regression z-scores derived

219 from actual and inferred expression within and between datasets. A one-sided Fisher's

220 exact test was used to compute the enrichment of prognostic TCGA IncRNAs (actual 
221 IncRNA expression) in the set of prognostic PRECOG IncRNAs (inferred IncRNA

222 expression). Prognostic IncRNAs were selected using FDR $<0.05$ and non-prognostic

223 IncRNAs were selected using a FDR $>0.1$. Protective (hazard ratio $<1$ ) and hazardous

224 (hazard ratio > 1) IncRNAs were analyzed using separate enrichment tests.

225

226 Revealing IncRNA-based subtypes in breast cancer

227 In the METABRIC dataset, feature selection was performed by selecting the top 500

228 IncRNAs with the highest variation of inferred expression across patients. The inferred

229 expression levels were then z-transformed across patients and gene-wise unsupervised

230 clustering was performed using Euclidean distance and complete linkage.

231

232 Analysis of prognostic and essential IncRNAs

233 Hazardous IncRNAs identified from the PRECOG meta-analysis of breast cancer and

234 hematopoietic cancer datasets were selected using a z-score cutoff of $>0$ and $p$-value

235 cutoff of $\leq 0.1$. IncRNA functional screening data were downloaded from Liu et al (Liu et

236 al., 2017) and contains averaged phenotype scores derived from systematic CRISPRi

237 knockout of IncRNAs. Essential IncRNAs are defined as those that when knocked down,

238 result in ablation of cell proliferation and cell death and is quantified by a phenotype

239 score included in the dataset. Essential IncRNAs in the MDA-MB-231 (breast) or K562

240 (hematopoietic) cell lines were selected using an average phenotype score cutoff of $<0$

241 and a $p$-value cutoff of $\leq 0.1$. The average phenotype score measures the growth effect

242 on the cell line when a particular IncRNA has been knocked down; a value $<0$ indicates

243 essentiality and a value $>0$ indicates non-essentiality. The enrichment overlap between 
244 essential MDA-MB-251 IncRNAs and hazardous breast cancer IncRNAs was computed

245 using a one-sided Fisher's exact test. The same test was used to calculate the

246 enrichment overlap between essential K562 IncRNAs and hazardous hematopoietic

247 cancer IncRNAs.

248

249 Prognostic IncRNAs and copy number alterations

250 IncRNAs associated with prognosis in the METABRIC dataset were mapped to the

251 genome for each patient. Hazardous IncRNAs were selected using z-score $>0$ and FDR

$252 \leq 0.01$ as the cutoff. Protective IncRNAs were selected using z-score $<0$ and FDR $\leq$

2530.01 as the cutoff. The copy number alteration (CNA) dataset provides the copy number

254 signal of genomic segments throughout the genome for each patient along with binary

255 calls indicating amplification (1) or deletion (-1). For each patient, a Fisher's exact test

256 was performed to measure significant enrichment of hazardous IncRNAs (compared to

257 protective IncRNAs) in genomic segments that had undergone copy number

258 amplification or deletion. When constructing the contingency table for a Fisher's exact

259 test, every cell had to have at least 5 counts in order for the test to be performed for the

260 patient to ensure robust enrichment results. In total, 1595 METABRIC patients were

261 used to test enrichment of hazardous IncRNAs in amplified regions and 901 patients

262 were used to test enrichment of protective IncRNAs in deleted regions. The Benjamini-

263 Hochberg procedure was used to adjust for multiple hypothesis testing. When

264 calculating the CNA signal corresponding to each IncRNA, the average copy number

265 signal of all segments overlapping the gene region (transcription start site to the

266 termination site) was used. 
When performing the CNA enrichment analysis in the TCGA dataset, IncRNAs

268

269

270

271

272

273

274

275

276

277

278

279

280

281

282

283

284

285

286

287

288

289

associated with prognosis in glioblastoma or ovarian cancer were selected using an

unadjusted p-value cutoff of $<0.05$. An FDR cutoff of 0.1 was used to identify prognostic

IncRNAs in pancreatic cancer and lung adenocarcinoma. These significance cutoffs

were chosen to ensure a sufficient number of prognostic IncRNAs for enrichment

analysis. Segments were selected using a CNA signal of $>0$ and $<0$ for amplification

and deletion, respectively.

\section{Results}

Overview of analysis

To systematically identify IncRNAs associated with patient prognosis, we applied the ARACNe-AP algorithm (Lachmann et al., 2016) to 23 TCGA RNA-seq datasets to generate IncRNA regulons for each cancer type. ARACNe-AP calculates the mutual information between a IncRNA and potential target genes and removes edges that are unlikely to represent a biological link using the concept of data processing inequality (Margolin et al., 2006; Lachmann et al., 2016). The resulting regulons represent a network where the edges encode the magnitude and direction of association between IncRNAs and other genes based on their gene expression across samples (See methods). A IncRNA's expression can be inferred within a microarray dataset lacking IncRNA probes by analyzing the aggregate expression of the protein coding genes composing that IncRNA's regulon. In total, we generated cancer-specific IncRNA regulons for 23 different cancer types using TCGA RNA-seq datasets. Once these regulons were generated, we transformed them into weight profiles and validated their 
290 predictive accuracy in TCGA. We then extended our analysis by inferring IncRNA

291 expression in microarray data compendia from PRECOG and METABRIC using the

292 regulon weight profiles and the BASE algorithm (Cheng et al., 2007). The BASE

293 algorithm outputs a predicted expression value for each IncRNA in every patient sample 294 by imputing the relative expression level of a IncRNA based on the expression of genes 295 that it correlates with (i.e. regulon genes). Regulon weight profiles were selected to 296 interrogate microarray data based on matched cancer type. After inferring the 297 expression of thousands of IncRNAs, we performed a systematic pan-cancer screen for 298 prognostic IncRNAs using survival information included in the microarray gene 299 expression data compendia (Figure 1).

300

301

Inferred IncRNA expression strongly correlates with actual expression

302 By implementing the ARACNe-AP algorithm in TCGA RNA-seq datasets, we 303 constructed thousands of IncRNA regulons for each TCGA cancer type (Figure 2A).

304 Each regulon contains a IncRNA and its associated genes, which can be used as 305 features to infer that specific IncRNA's expression. An example of an inferred IncRNA 306 expression pattern and the expression of its associated genes is provided in Figure $\mathbf{S 1 .}$

307 The number of regulons varied across cancer types depending on whether any genes 308 were found to have high mutual information with any given IncRNA based on expression signal. To confirm that the inferred expression of the IncRNAs was indeed accurate, we

310 correlated each IncRNA's inferred expression with its actual expression in all TCGA

311 RNA-seq datasets. We observed that for the majority of IncRNAs, their inferred and

312 actual expression were highly correlated across 23 cancer types as shown by the left- 
313 skewed distribution of correlation coefficients (Figure 2B). These results indicate that it

314 is possible to infer the expression levels of IncRNAs based on the aggregate expression

315 of its associated genes.

316 Furthermore, we inferred IncRNA expression in the METABRIC dataset and

317 compared the inferred and actual expression of 95 IncRNAs, which had probes present

318 in the microarray platform. We observed that 82 of these IncRNAs had inferred

319 expression values positively correlated with probe expression with 59 having significant

320 associations (Figure 3A; $p \leq 0.05$ ). As an example, the correlation between the inferred

321 and actual expression of HOTAIR and PVT1 was 0.54 and 0.60 , respectively (Figure

322 3B-C). This analysis was repeated in each PRECOG dataset and we again observed

323 that the correlation coefficient distributions were left-skewed indicating that

324 approximately $95 \%$ of the inferred IncRNA expression values were positively correlated

325 with actual probe expression (Figure 3D-F), with a median correlation coefficient of 0.6.

326 These results demonstrate that IncRNA expression can be inferred using the expression

327 of protein coding genes in microarray datasets. Furthermore, we show our IncRNA

328 inference platform is robust and can be generalized to different datasets as

329 demonstrated by our analysis of TCGA, PRECOG, and METABRIC.

330 Previous studies have shown that the expression patterns of IncRNAs

331 recapitulate the four well-known molecular subtypes in breast cancer (Su et al., 2014),

332 which are associated with tumor behavior and patient prognosis (Perou et al., 2000).

333 We sought to confirm whether inferred IncRNA expression could similarly distinguish

334 between the different breast cancer subtypes. Using the METABRIC dataset, we 335 performed hierarchical clustering of the genes using the inferred expression values of 
336500 IncRNAs with highest variance across samples and indeed found subtype-specific

337 differences in inferred IncRNA expression (Figure 3G). This finding implies that IncRNA

338 activity varies across breast cancer molecular subtypes and may play a role in tumor

339 behavior.

340

341 Exploring the prognostic landscape of IncRNAs across 20 tumor types

342 In light of the current dearth of RNA-seq datasets with survival metadata and the

343 expansive trove of microarray datasets that do have this valuable clinical information,

344 we first used the PRECOG compendia to systematically infer IncRNA expression.

345 Datasets in PRECOG all include patient survival information, and many patients within

346 these datasets have been followed-up for longer periods of time compared to those

347 recorded in TCGA, offering greater statistical power when performing survival analyses

348 (Clark et al., 2003). We carried out a systematic inference of prognostic IncRNAs in

349 PRECOG datasets with a sufficient number of probes (500) and performed a meta-

350 analysis by combining the results within each tissue type. From this systematic screen,

351 we identified a number of prognostic IncRNAs associated with both increased and

352 decreased patient mortality risk in 13 PRECOG cancer types (Figure 4A). These results

353 suggest that IncRNAs may play a substantial role in the progression of all neoplasia. An

354 overview of all IncRNAs and their associations with prognosis is available in the

355 Supplementary Results.

356 Furthermore, we found that some IncRNAs including HOTAIR and H19 were

357 associated with poor survival across multiple cancer types (Figure 4A). These results

358 are in accordance with several reports implicating these IncRNAs in neoplastic 
359 progression and metastasis across different cancer types (Matouk et al., 2007; Gupta et

360 al., 2010). It has been suggested that HOTAIR promotes cancer invasiveness and

361 metastasis by the induction of a more embryonic-like state (Gupta et al., 2010), which

362 leads to increased resistance to known therapies and is a marker for poor prognosis in

363 almost all cancer types (Ge et al., 2017; Shibue \& Weinberg, 2017). However, some

364 IncRNAs like TINCR were associated with both good and poor prognosis depending on

365 the cancer type (Figure 4B). Particularly, TINCR was associated with unfavorable

366 prognosis in breast cancer, which is consistent with the implication of TINCR in

367 promoting breast cancer tumorigenesis (Xu et al., 2017; Liu et al., 2018). TINCR can

368 stabilize mRNA by preventing Staufen-mediated mRNA decay of differentiation genes in

369 epidermal tissue (Kretz et al., 2013), but it is unclear whether this mechanism plays a

370 role in tumor evolution.

371 To provide evidence that the identified prognostic IncRNAs are functionally

372 relevant, we performed an integrative analysis of CRISPRi data generated from a high-

373 throughput, systematic screen for IncRNAs that are essential for cancer cell growth (Liu

374 et al., 2017). We calculated the enrichment of functional IncRNAs identified in MDA-MB-

375231 and K562 cell lines in the set of prognostic IncRNAs identified in breast and

376 hematopoietic cancers, respectively. We observed that hazardous IncRNAs in

377 hematopoietic cancers were enriched in essential IncRNAs (Odds ratio $=2.26, p=7.1 \mathrm{e}-$

3785 ) and protective IncRNAs were depleted in essential IncRNAs (Odds ratio $=0.73, p=$

3790.16 ) indicating that hazardous IncRNAs in hematopoietic cancers tend to be required

380 for cancer cell growth compared to protective or non-prognostic IncRNAs (Figure 4C-

381 D). Likewise, we discovered that hazardous IncRNAs in breast cancer was also 
382 enriched in essential IncRNAs (Odds ratio $=3.04, p=5.4 \mathrm{e}-3$ ) and protective IncRNAs

383 were depleted in essential IncRNAs (Odds ratio $=0.30, p=3.2 e-4$ ), again suggesting

384 that hazardous IncRNAs are more likely to be essential because they contribute to cell

385 growth and are thus associated with increased mortality risk (Figure 4E-F). Together,

386 these results indicate that hazardous IncRNAs identified in our analysis of breast and

387 hematopoietic cancers are functionally relevant, at least in the context of in vitro cancer 388 cell growth.

389 In addition to known cancer-associated IncRNAs, our screen also generated

390 novel hypotheses about IncRNAs that have not been well-studied in certain cancer

391 types. To highlight, high TINCR inferred expression was associated with improved

392 survival in patients with brain tumors (Figure 4G, $\mathrm{p}=5 \mathrm{e}-8$ ). Moreover, high H19 inferred

393 expression was associated with decreased mortality risk among patients with liver

394 tumors (Figure 4H, $p=0.002$ ). EGOT inferred expression was associated with

395 decreased mortality risk in patients with breast cancer (Figure 4I, $p=9 e-11$ ). This is

396 consistent with a previous study, which showed that downregulation of EGOT correlates

397 with worse clinicopathological features and poor prognosis in breast cancer (Xu et al.,

398 2015). Lastly, we found that high inferred RP11-108P20.4 expression was associated

399 with improved survival in prostate cancer (Figure 4J, $p=2 e-15$ ), which coincides with a

400 recent report introducing RP11-108P20.4 as part of a four IncRNA gene prognostic risk

401 signature for prostate cancer (Huang et al., 2002). These results demonstrate that novel

402 prognostic IncRNAs can be identified across several cancer types from common

403 microarray datasets. 
Furthermore, to assess the reproducibility of our screen, we performed a survival

405

406

407

408

409

410

411

412

413

414

415

416

417

418

419

420

421

422

423

424

425

426

analysis of 23 TCGA cancer types to identify prognostic IncRNAs using their actual

expression in each dataset. From this screen, prognostic IncRNAs (FDR $<0.05)$ were identified in 5 cancer types (LUAD, LGG, BLCA, LIHC, and LAML). We stratified the IncRNAs into protective or hazardous, and computed their enrichment, respectively, in protective or hazardous IncRNAs predicted from the PRECOG datasets. We identified significant overlap between the two sets of prognostic IncRNAs in all 5 cancer types

(Figure 5A). Moreover, we compared the Cox regression z-scores (TCGA) and meta zscores (PRECOG) for all IncRNAs within lung adenocarcinoma, low-grade glioma, and bladder cancer datasets, and observed significant correlations (Figure 5B-D). These zscores were calculated by dividing the Cox regression coefficient by its standard error and the meta z-scores were calculated using weighted Stouffer's z-score method using the dataset sample size as weights. Several well-studied IncRNAs including $\mathrm{H} 19$, BCAR4, GAS5, XIST, HOTAIR, and EGOT had concordant z-scores (Figure 5B-D).

IncRNAs associated with prognosis localize to genomic regions under selective pressure

Operating under the hypothesis that genomic amplifications and deletions indicate regions of positive and negative selective pressure by the tumor, respectively (Zack et al., 2013), we aimed to provide further evidence that IncRNAs associated with prognosis are also linked to genomic structural abnormalities that confer a selective advantage to neoplastic cells. Thus, we analyzed copy number alteration together with inferred IncRNA expression of each patient sample in the METABRIC data set. Strikingly, we 
427 observed a significant enrichment of IncRNAs associated with poor prognosis

428 (hazardous) in amplified regions of the genome in 448 METABRIC patient tumors

429 (Figure 6A). In comparison, we only observed 54 patient tumors where amplified

430 regions were significantly depleted of hazardous IncRNAs. (Figure 6B). Likewise, we

431 observed 47 patients with deleted genomic regions enriched in IncRNAs associated with

432 decreased mortality (protective), compared to 17 patients who had protective IncRNAs

433 depleted in deleted genomic regions. We also explored whether prognostic IncRNAs

434 were enriched in amplified or deleted regions of the genome in pancreatic cancer, lung

435 adenocarcinoma, and glioblastoma TCGA datasets and observed consistent results

436 (Figure S2). In summary, these results indicate that prognostic IncRNAs localize to

437 genomic regions that undergo copy number alteration suggesting that they are under

438 both positive and negative selective pressure by the tumor.

439 To demonstrate that IncRNAs associated with mortality risk are under selection,

440 we highlight JRK and CADM3-AS1. In our analysis of JRK, we discovered that patients

441 with high inferred JRK expression exhibited a higher mortality rate compared to patients

442 with low inferred JRK expression (Figure 6C). In conjunction with this result, we also

443 observed higher amplification signal of the region harboring JRK in patients with high

444 inferred JRK expression compared to patients with low inferred JRK expression (Figure

445 6D). These results suggest that JRK exhibits a pro-oncogenic effect because it is under

446 positive selection by breast tumors, which consistently coincides with their association

447 with increased mortality risk. In contrast, we found that high CADM3-AS1 inferred

448 expression was associated with a more favorable prognosis compared to patients with

449 low CADM3-AS1 inferred expression (Figure 6E). In agreement with our prediction, we 
450 found that the genomic region harboring CADM3-AS1 was significantly more amplified

451 in patients with low CADM3-AS1 expression compared to patients with high CADM3-

452 AS1 expression (Figure 6F). Together, these results suggest that due to CADM3-AS1's

453 association with decreased mortality risk, it exhibits anti-tumor effects that are not

454 selected for by breast neoplasms. Our analysis of prognostic IncRNAs in the context of

455 copy number alteration indicates that they are under selective pressure and provides

456 evidence that they are functionally involved in cancer development. This hypothesis

457 compliments a recent publication showing that somatic copy number variations in

458 IncRNA loci were predictive of target gene expression and might be responsible for the 459 dysregulation of dozens of cancer-associated genes (Chiu et al., 2018).

460

461

462 Discussion

463 Investigation into IncRNAs using integrative and systematic approaches can help

464 provide insight into the genome's "dark matter" and how it may influence disease and

465 ultimately patient prognosis in cancer. Studies are now underway to characterize and

466 dissect the intricacies of IncRNA regulatory mechanisms in several biological contexts

467 which may revise our current understanding of genome regulation (Cech \& Steitz,

468 2014). Hypothesis generating projects are essential for guiding the biomedical

469 community towards investigating more promising leads as to accelerate the discovery of

470 novel drug targets and biomarkers for cancer and other diseases. We have proposed a

471 novel analysis framework to infer IncRNA expression in microarray gene expression

472 data compendia and subsequently carry out systematic survival analysis to identify 
473 prognostic IncRNAs across 20 cancer types. Our approach is novel in that we utilize

474 expression information from TCGA RNA-seq data to generate cancer-specific IncRNA

475 regulon profiles that capture the IncRNA-gene relationships within a specific tissue

476 context. We then apply these profiles to microarray gene expression data using a

477 sensitive enrichment algorithm, BASE, to infer IncRNA expression based primarily on

478 protein coding gene expression. We perform this analysis at a pan-cancer scale to

479 identify new prognostic IncRNAs that have global and tissue-specific associations with

480 survival. In contrast to other prognostic IncRNA pan-cancer analyses, we evaluated

481 IncRNA expression in a large number of microarray datasets, providing us with a more

482 comprehensive view of prognostic IncRNAs in cancer.

483 In particular, we were able to validate that the inferred IncRNA expression values

484 are accurate and reproducible within and across several datasets. We identified novel

485 associations between IncRNAs and patient mortality risk across 13 (out of 20 total)

486 cancer types that can be further evaluated in more detail. We confirmed that

487 associations between IncRNA expression and mortality risk were consistent regardless

488 of whether inferred or actual expression were used and showed that prognostic

489 IncRNAs identified in breast and hematopoietic cancers were significantly enriched in

490 functional IncRNAs required for cell growth. Furthermore, we demonstrated that

491 hazardous IncRNAs were enriched within regions under positive selective pressure.

492 In spite of the evidence we provide, this study does have limitations that are

493 imposed by the data. First, in each microarray dataset, we used the regulon profile that

494 was generated from the TCGA cancer type that was the best match based on tissue.

495 However, it was not always possible to find an exact cancer type match for each 
496 microarray dataset. Thus, using an inappropriately matched regulon profile may yield

497 false associations. Second, univariate Cox regression models were used to screen for

498 prognostic IncRNAs, which do not account for other clinical or demographic factors that

499 may modify the associations. These may include age, gender, race, histological marker

500 status, stage, and grade. However, as an initial screen our framework can be further

501 improved to include multivariate analyses in follow up studies if more specific

502 hypotheses are to be tested (McNamee, 2005). Third, not all IncRNAs are poly-

503 adenylated and are thus captured in poly-(A)-enriched RNA-seq or microarray studies.

504 Due to this, we likely did not include all known IncRNAs in our study. Lastly, our analysis

505 does not account for all cancer subtypes to address the issue of molecular

506 heterogeneity within the same cancer type (Gerdes et al., 2014). As a result, certain

507 associations between IncRNA expression and prognosis may only be valid in certain

508 subtypes of the same cancer type. As stated previously, future analyses can address

509 this issue by performing subgroup analyses within specific subtypes.

510

\section{Conclusions}

512 Our approach can be extended to other microarray gene expression datasets by

513 utilizing our regulon profiles to infer IncRNA expression. As a result, it is possible to

514 identify novel associations between IncRNAs and other disease phenotypes other than

515 survival. Moreover, is possible to generate regulon profiles for other non-coding RNA

516 species and infer their expression in microarray datasets. In summary, our systematic

517 analysis introduces new avenues to investigate clinically relevant IncRNAs and 
518 demonstrate that these long, diverse transcripts constitute a new source of gene

519 products that can serve as novel drug targets or biomarkers.

520

521 Authors Contributions

522 M.U. and C.C. conceived the project. All authors performed computational analyses.

523 M.U., E.S., and C.C. wrote the manuscript. All authors critically reviewed the content. All

524 authors read and approved the final manuscript.

525

526

\section{References}

527

528

529

530

531

532

533

534

535

536

537

538

539

540

541

542

543

544

545

546

547

548

549

550

551

552

553

Ali MM, Akhade VS, Kosalai ST, Subhash S, Statello L, Meryet-Figuiere M, Abrahamsson J, Mondal T, Kanduri C. 2018. PAN-cancer analysis of S-phase enriched IncRNAs identifies oncogenic drivers and biomarkers. Nature Communications 9. DOI: 10.1038/s41467-018-03265-1.

Alvarez MJ, Shen Y, Giorgi FM, Lachmann A, Ding BB, Ye BH, Califano A. 2016. Functional characterization of somatic mutations in cancer using network-based inference of protein activity. Nature Genetics 48:838-847. DOI: 10.1038/ng.3593.

Ashouri A, Sayin VI, Van den Eynden J, Singh SX, Papagiannakopoulos T, Larsson E. 2016. Pan-cancer transcriptomic analysis associates long non-coding RNAs with key mutational driver events. Nature Communications 0. DOI: 10.1038/ncomms13197.

Bartonicek N, Maag JLV, Dinger ME. 2016. Long noncoding RNAs in cancer: mechanisms of action and technological advancements. Molecular Cancer 15:43. DOI: 10.1186/s12943-016-0530-6.

Byron SA, Keuren-Jensen KRV, Engelthaler DM, Carpten JD, Craig DW. 2016. Translating RNA sequencing into clinical diagnostics: opportunities and challenges. Nature Reviews Genetics 17:257-271. DOI: 10.1038/nrg.2016.10.

Cabanski CR, White NM, Dang HX, Silva-Fisher JM, Rauck CE, Cicka D, Maher CA. 2015. Pan-cancer transcriptome analysis reveals long noncoding RNAs with conserved function. RNA biology 12:628-642. DOI: 10.1080/15476286.2015.1038012.

Cech TR, Steitz JA. 2014. The Noncoding RNA Revolution-Trashing Old Rules to Forge New Ones. Cell 157:77-94. DOI: 10.1016/j.cell.2014.03.008.

Cheng C, Yan X, Sun F, Li LM. 2007. Inferring activity changes of transcription factors by binding association with sorted expression profiles. BMC Bioinformatics 8:452. DOI: 10.1186/1471-2105-8-452. 
554

555

556

557

558

559

560

561

562

563

564

565

566

567

568

569

570

571

572

573

574

575

576

577

578

579

580

581

582

583

584

585

586

587

588

589

590

591

592

593

594

595

596

597

598

599

Ching T, Peplowska K, Huang S, Zhu X, Shen Y, Molnar J, Yu H, Tiirikainen M, Fogelgren B, Fan R, Garmire LX. 2016. Pan-Cancer Analyses Reveal Long Intergenic Non-Coding RNAs Relevant to Tumor Diagnosis, Subtyping and Prognosis. EBioMedicine 7:62-72. DOI: 10.1016/j.ebiom.2016.03.023.

Chiu H-S, Somvanshi S, Patel E, Chen T-W, Singh VP, Zorman B, Patil SL, Pan Y, Chatterjee SS, Cancer Genome Atlas Research Network, Sood AK, Gunaratne $\mathrm{PH}$, Sumazin P. 2018. Pan-Cancer Analysis of IncRNA Regulation Supports Their Targeting of Cancer Genes in Each Tumor Context. Cell Reports 23:297312.e12. DOI: 10.1016/j.celrep.2018.03.064.

Clark TG, Bradburn MJ, Love SB, Altman DG. 2003. Survival analysis part I: basic concepts and first analyses. British Journal of Cancer 89:232-238. DOI: 10.1038/sj.bjc.6601118.

Consortium TEP. 2012. An integrated encyclopedia of DNA elements in the human genome. Nature 489:57-74. DOI: 10.1038/nature11247.

Curtis C, Shah SP, Chin S-F, Turashvili G, Rueda OM, Dunning MJ, Speed D, Lynch AG, Samarajiwa S, Yuan Y, Gräf S, Ha G, Haffari G, Bashashati A, Russell R, McKinney S, METABRIC Group, Langerød A, Green A, Provenzano E, Wishart G, Pinder S, Watson P, Markowetz F, Murphy L, Ellis I, Purushotham A, Børresen-Dale A-L, Brenton JD, Tavaré S, Caldas C, Aparicio S. 2012. The genomic and transcriptomic architecture of 2,000 breast tumours reveals novel subgroups. Nature 486:346-352. DOI: 10.1038/nature10983.

Du Z, Fei T, Verhaak RGW, Su Z, Zhang Y, Brown M, Chen Y, Liu XS. 2013. Integrative genomic analyses reveal clinically relevant long noncoding RNAs in human cancer. Nature Structural \& Molecular Biology 20:908-913. DOI: 10.1038/nsmb.2591.

Esteller M. 2011. Non-coding RNAs in human disease. Nature Reviews. Genetics 12:861-874. DOI: 10.1038/nrg3074.

Evans JR, Feng FY, Chinnaiyan AM. 2016. The bright side of dark matter: IncRNAs in cancer. The Journal of Clinical Investigation 126:2775-2782. DOI: 10.1172/JCl84421.

Ge Y, Gomez NC, Adam RC, Nikolova M, Yang H, Verma A, Lu CP-J, Polak L, Yuan S, Elemento O, Fuchs E. 2017. Stem Cell Lineage Infidelity Drives Wound Repair and Cancer. Cell 169:636-650.e14. DOI: 10.1016/j.cell.2017.03.042.

Gentles AJ, Newman AM, Liu CL, Bratman SV, Feng W, Kim D, Nair VS, Xu Y, Khuong A, Hoang CD, Diehn M, West RB, Plevritis SK, Alizadeh AA. 2015. The prognostic landscape of genes and infiltrating immune cells across human cancers. Nature Medicine 21:938-945. DOI: 10.1038/nm.3909.

Gerdes MJ, Sood A, Sevinsky C, Pris AD, Zavodszky MI, Ginty F. 2014. Emerging understanding of multiscale tumor heterogeneity. Frontiers in Oncology 4:366. DOI: 10.3389/fonc.2014.00366.

Guo L, Yao L, Jiang Y. 2016. A novel integrative approach to identify IncRNAs associated with the survival of melanoma patients. Gene 585:216-220. DOI: 10.1016/j.gene.2016.03.036.

Gupta RA, Shah N, Wang KC, Kim J, Horlings HM, Wong DJ, Tsai M-C, Hung T, Argani P, Rinn JL, Wang Y, Brzoska P, Kong B, Li R, West RB, van de Vijver MJ, Sukumar S, Chang HY. 2010. Long non-coding RNA HOTAIR reprograms

Peer) reviewing PDF | (2019:11:43305:2:0:NEW 10 Feb 2020) 
600

601

602

603

604

605

606

607

608

609

610

611

612

613

614

615

616

617

618

619

620

621

622

623

624

625

626

627

628

629

630

631

632

633

634

635

636

637

638

639

640

641

642

643

644

chromatin state to promote cancer metastasis. Nature 464:1071-1076. DOI: 10.1038/nature08975.

Gutschner T, Diederichs S. 2012. The hallmarks of cancer: a long non-coding RNA point of view. RNA biology 9:703-719. DOI: 10.4161/rna.20481.

Hanahan D, Weinberg RA. 2000. The hallmarks of cancer. Cell 100:57-70.

Huang K-C, Rao PH, Lau CC, Heard E, Ng S-K, Brown C, Mok SC, Berkowitz RS, Ng S-W. 2002. Relationship of XIST expression and responses of ovarian cancer to chemotherapy. Molecular Cancer Therapeutics 1:769-776.

lyer MK, Niknafs YS, Malik R, Singhal U, Sahu A, Hosono Y, Barrette TR, Prensner JR, Evans JR, Zhao S, Poliakov A, Cao X, Dhanasekaran SM, Wu Y-M, Robinson DR, Beer DG, Feng FY, lyer HK, Chinnaiyan AM. 2015. The landscape of long noncoding RNAs in the human transcriptome. Nature Genetics 47:199-208. DOI: 10.1038/ng.3192.

Kretz M, Siprashvili Z, Chu C, Webster DE, Zehnder A, Qu K, Lee CS, Flockhart RJ, Groff AF, Chow J, Johnston D, Kim GE, Spitale RC, Flynn RA, Zheng GXY, Aiyer S, Raj A, Rinn JL, Chang HY, Khavari PA. 2013. Control of somatic tissue differentiation by the long non-coding RNA TINCR. Nature 493:231-235. DOI: 10.1038/nature11661.

Kung JTY, Colognori D, Lee JT. 2013. Long noncoding RNAs: past, present, and future. Genetics 193:651-669. DOI: 10.1534/genetics.112.146704.

Lachmann A, Giorgi FM, Lopez G, Califano A. 2016. ARACNe-AP: gene network reverse engineering through adaptive partitioning inference of mutual information. Bioinformatics (Oxford, England) 32:2233-2235. DOI: 10.1093/bioinformatics/btw216.

Li J, Han L, Roebuck P, Diao L, Liu L, Yuan Y, Weinstein JN, Liang H. 2015. TANRIC: An Interactive Open Platform to Explore the Function of IncRNAs in Cancer. Cancer Research 75:3728-3737. DOI: 10.1158/0008-5472.CAN-15-0273.

Liu Y, Du Y, Hu X, Zhao L, Xia W. 2018. Up-regulation of ceRNA TINCR by SP1 contributes to tumorigenesis in breast cancer. BMC cancer 18:367. DOI: 10.1186/s12885-018-4255-3.

Liu SJ, Horlbeck MA, Cho SW, Birk HS, Malatesta M, He D, Attenello FJ, Villalta JE, Cho MY, Chen Y, Mandegar MA, Olvera MP, Gilbert LA, Conklin BR, Chang HY, Weissman JS, Lim DA. 2017. CRISPRi-based genome-scale identification of functional long noncoding RNA loci in human cells. Science (New York, N.Y.) 355. DOI: 10.1126/science.aah7111.

Love MI, Huber W, Anders S. 2014. Moderated estimation of fold change and dispersion for RNA-seq data with DESeq2. Genome Biology 15:550. DOI: 10.1186/s13059-014-0550-8.

Margolin AA, Nemenman I, Basso K, Wiggins C, Stolovitzky G, Favera RD, Califano A. 2006. ARACNE: An Algorithm for the Reconstruction of Gene Regulatory Networks in a Mammalian Cellular Context. BMC Bioinformatics 7:S7. DOI: 10.1186/1471-2105-7-S1-S7.

Matouk IJ, DeGroot N, Mezan S, Ayesh S, Abu-lail R, Hochberg A, Galun E. 2007. The $\mathrm{H} 19$ non-coding RNA is essential for human tumor growth. PloS One 2:e845. DOI: 10.1371/journal.pone.0000845.

Peer] reviewing PDF | (2019:11:43305:2:0:NEW 10 Feb 2020) 
645

646

647

648

649

650

651

652

653

654

655

656

657

658

659

660

661

662

663

664

665

666

667

668

669

670

671

672

673

674

675

676

677

678

679

680

681

682

683

684

685

686

687

688

689

690
Mattick JS, Makunin IV. 2006. Non-coding RNA. Human Molecular Genetics 15 Spec No 1:R17-29. DOI: $10.1093 / \mathrm{hmg} / \mathrm{ddl0} 46$.

McNamee R. 2005. Regression modelling and other methods to control confounding. Occupational and Environmental Medicine 62:500-506. DOI: 10.1136/oem.2002.001115.

Perou CM, Sørlie T, Eisen MB, van de Rijn M, Jeffrey SS, Rees CA, Pollack JR, Ross DT, Johnsen H, Akslen LA, Fluge O, Pergamenschikov A, Williams C, Zhu SX, Lønning PE, Børresen-Dale AL, Brown PO, Botstein D. 2000. Molecular portraits of human breast tumours. Nature 406:747-752. DOI: 10.1038/35021093.

Ponting CP, Oliver PL, Reik W. 2009. Evolution and functions of long noncoding RNAs. Cell 136:629-641. DOI: 10.1016/j.cell.2009.02.006.

Rinn JL, Chang HY. 2012. Genome regulation by long noncoding RNAs. Annual Review of Biochemistry 81:145-166. DOI: 10.1146/annurev-biochem-051410-092902.

Sahu A, Singhal U, Chinnaiyan AM. 2015. Long noncoding RNAs in cancer: from function to translation. Trends in Cancer 1:93-109. DOI: 10.1016/j.trecan.2015.08.010.

Schalken, J.A. de K JB. 2002. DD3 (PCA3), a Very Sensitive and Specific Marker to Detect Prostate Tumors. 62:2695-2698.

Schmitt AM, Chang HY. 2016. Long Noncoding RNAs in Cancer Pathways. Cancer Cell 29:452-463. DOI: 10.1016/j.ccell.2016.03.010.

Shibue T, Weinberg RA. 2017. EMT, CSCs, and drug resistance: the mechanistic link and clinical implications. Nature Reviews. Clinical Oncology 14:611-629. DOI: 10.1038/nrclinonc.2017.44.

Su X, Malouf GG, Chen Y, Zhang J, Yao H, Valero V, Weinstein JN, Spano J-P, MericBernstam F, Khayat D, Esteva FJ. 2014. Comprehensive analysis of long noncoding RNAs in human breast cancer clinical subtypes. Oncotarget 5:98649876.

Wang KC, Chang HY. 2011. Molecular mechanisms of long noncoding RNAs. Molecular Cell 43:904-914. DOI: 10.1016/j.molcel.2011.08.018.

Xu S, Kong D, Chen Q, Ping Y, Pang D. 2017. Oncogenic long noncoding RNA landscape in breast cancer. Molecular Cancer 16:129. DOI: 10.1186/s12943017-0696-6.

Xu S-P, Zhang J-F, Sui S-Y, Bai N-X, Gao S, Zhang G-W, Shi Q-Y, You Z-L, Zhan C, Pang D. 2015. Downregulation of the long noncoding RNA EGOT correlates with malignant status and poor prognosis in breast cancer. Tumour Biology: The Journal of the International Society for Oncodevelopmental Biology and Medicine 36:9807-9812. DOI: 10.1007/s13277-015-3746-y.

Yan X, Hu Z, Feng Y, Hu X, Yuan J, Zhao SD, Zhang Y, Yang L, Shan W, He Q, Fan L, Kandalaft LE, Tanyi JL, Li C, Yuan C-X, Zhang D, Yuan H, Hua K, Lu Y, Katsaros D, Huang Q, Montone K, Fan Y, Coukos G, Boyd J, Sood AK, Rebbeck T, Mills GB, Dang CV, Zhang L. 2015. Comprehensive Genomic Characterization of Long Non-coding RNAs across Human Cancers. Cancer Cell 28:529-540. DOI: 10.1016/j.ccell.2015.09.006.

Zack TI, Schumacher SE, Carter SL, Cherniack AD, Saksena G, Tabak B, Lawrence MS, Zhsng C-Z, Wala J, Mermel CH, Sougnez C, Gabriel SB, Hernandez B, Shen H, Laird PW, Getz G, Meyerson M, Beroukhim R. 2013. Pan-cancer 
691 patterns of somatic copy number alteration. Nature Genetics 45:1134-1140. DOI: $692 \quad 10.1038 / \mathrm{ng} .2760$.

693 


\section{Figure 1}

Overview of analysis.

TCGA RNA-seq data from 23 cancer types were used as input into the ARACNe algorithm to generate cancer type specific IncRNA-target gene regulons. These regulons were used with the BASE algorithm to infer IncRNA expression in PRECOG and METABRIC microarray datasets. The BASE algorithm infers the expression of IncRNAs in microarray data using the aggregate expression of the IncRNAs' associated protein coding genes. Lastly, a systematic pan-cancer analysis of 9,463 IncRNAs was carried out to identify prognostic IncRNAs across 20 different cancer types in the microarray data compendia. 


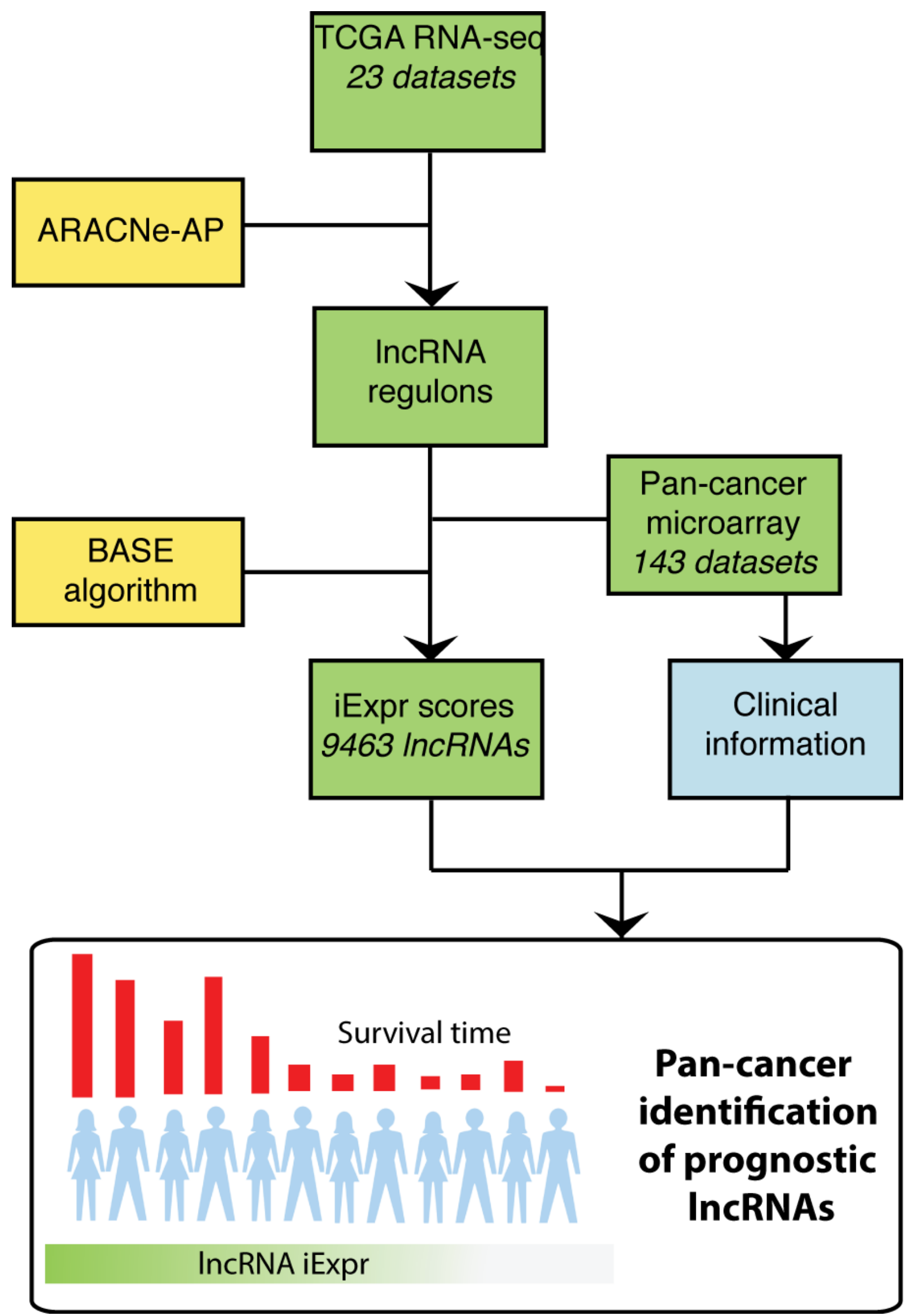


Figure 2

Comparison of inferred IncRNA expression and actual IncRNA expression.

(A) Number of IncRNA regulons identified in 23 TCGA cancer types from the ARACne algorithm. Each regulon consists of a IncRNA and its associated protein coding genes. (B) Distribution of Spearman correlation coefficients from comparing inferred IncRNA expression with its actual expression using RNA-seq data from 23 TCGA cancer types. 


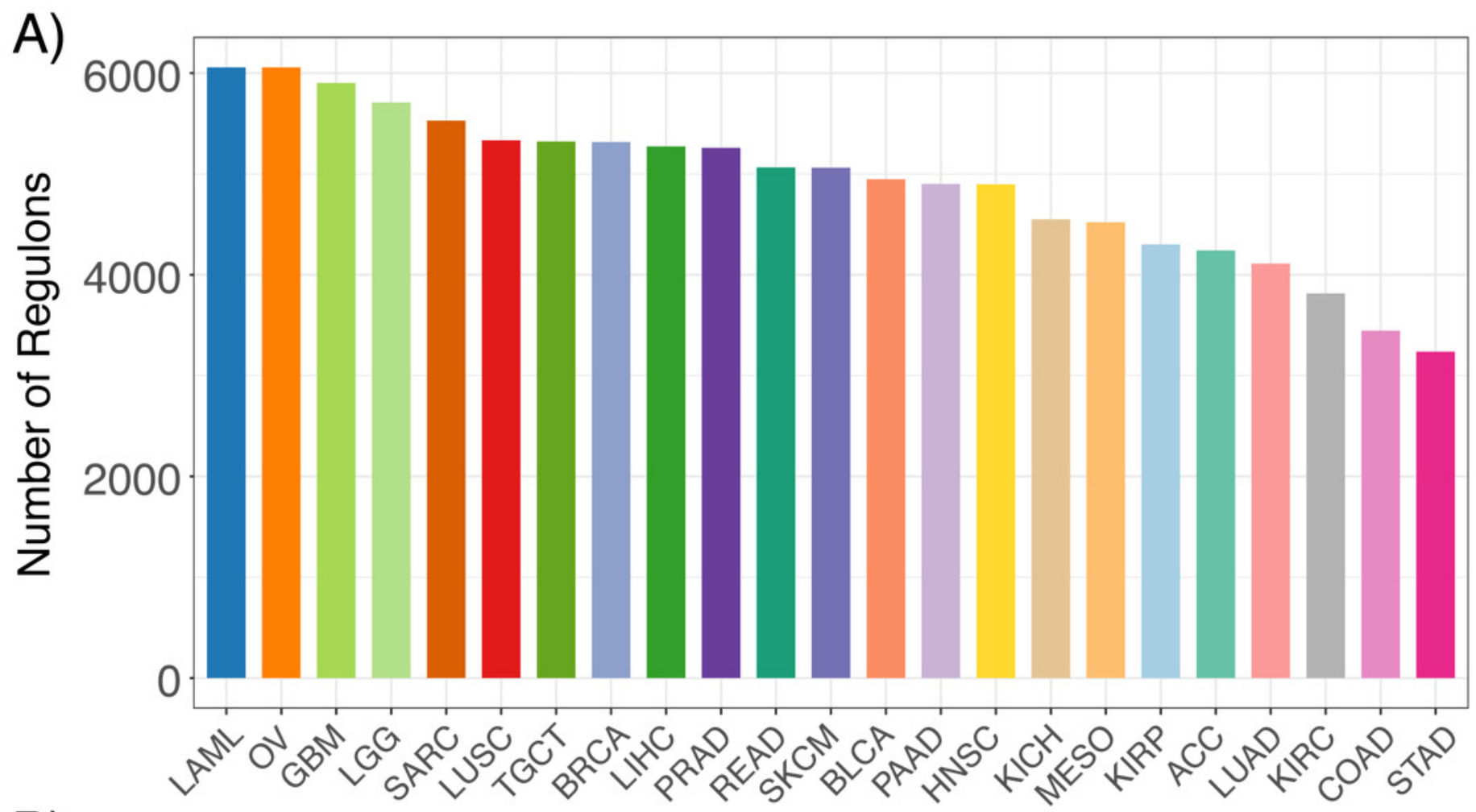

B)

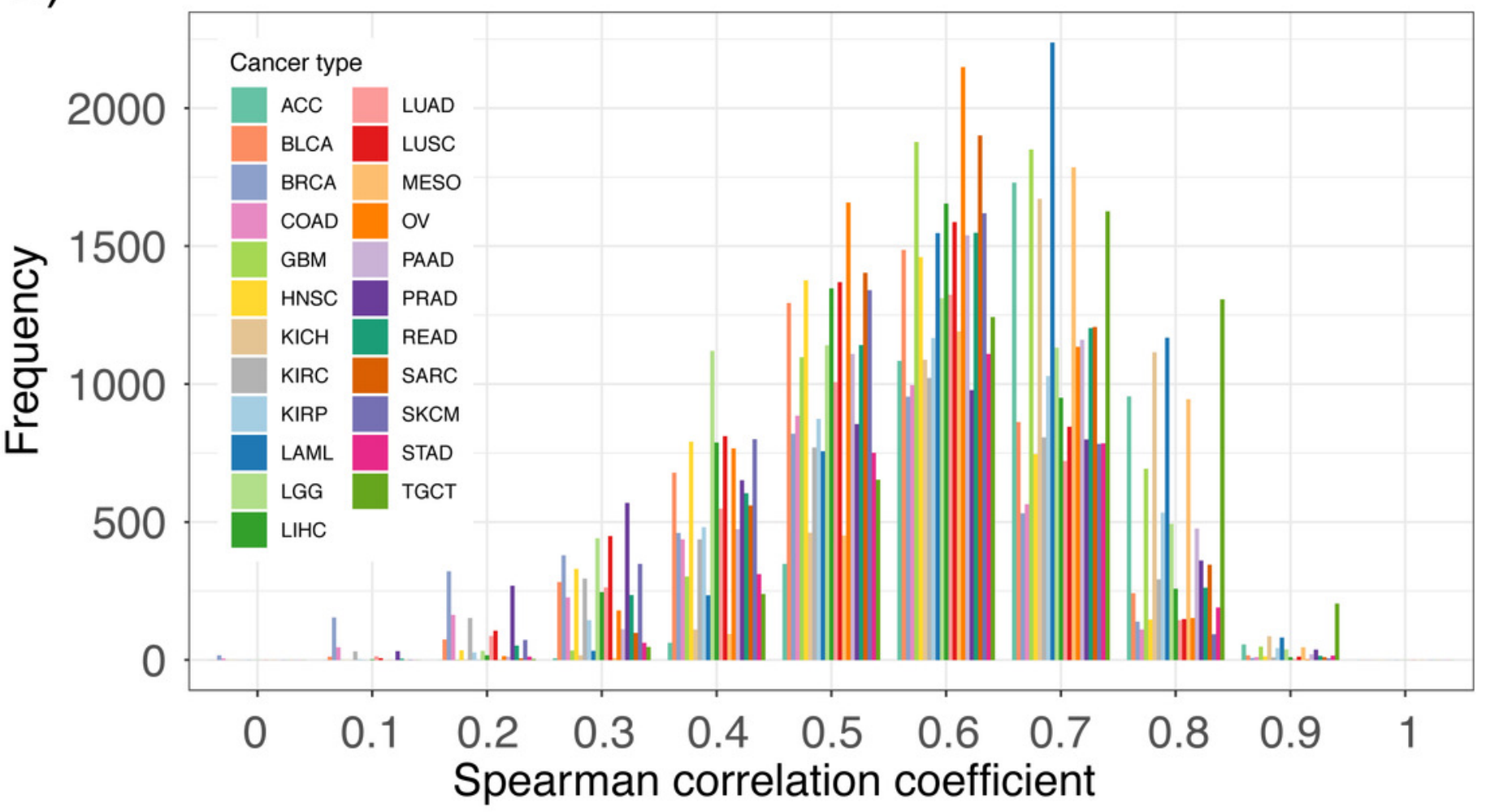




\section{Figure 3}

Comparison of inferred and actual IncRNA expression in METABRIC using available probes.

(A) Waterfall plot showing correlation of inferred IncRNA expression and IncRNA probe expression in the METABRIC microarray dataset. Each IncRNA that had an available probe in the METABRIC microarray platform was selected to compare its inferred expression with its actual expression using Spearman correlation. Scatterplots show correlation of inferred and actual expression for (B) HOTAIR and (C) PVT1. (D-F) Distribution of correlation coefficients between inferred IncRNA expression and actual probe expression for 141 microarray datasets across 20 cancer types in the PRECOG compendium. Dashed vertical line indicates no correlation. Panels separated to increase legibility. (G) Heatmap showing inferred IncRNA expression differences between Luminal A, Luminal B, Normal-like, HER2-enriched, and Basal breast cancer subtypes. Color bar shows z-score spectrum. 
A)

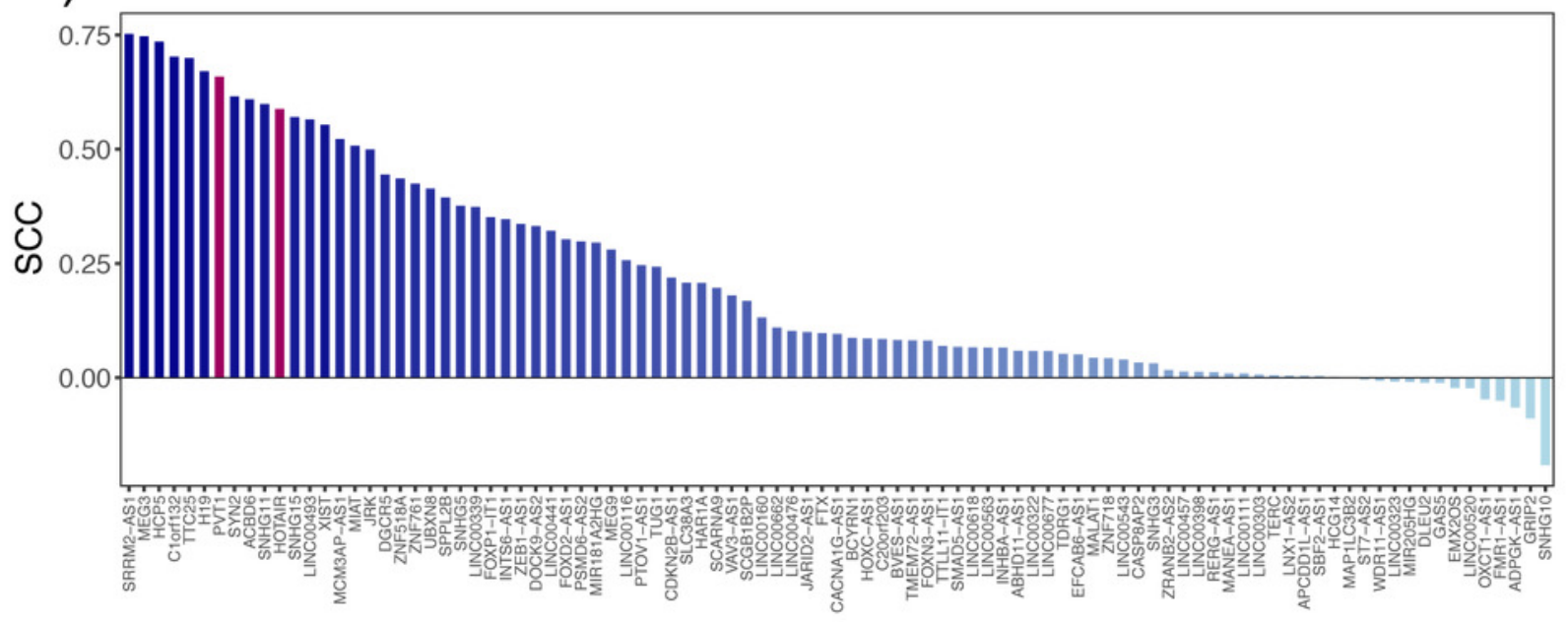

B)

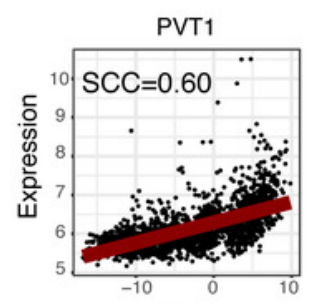

C)

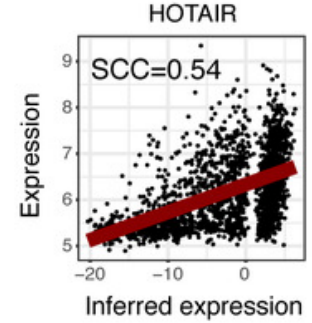

D)

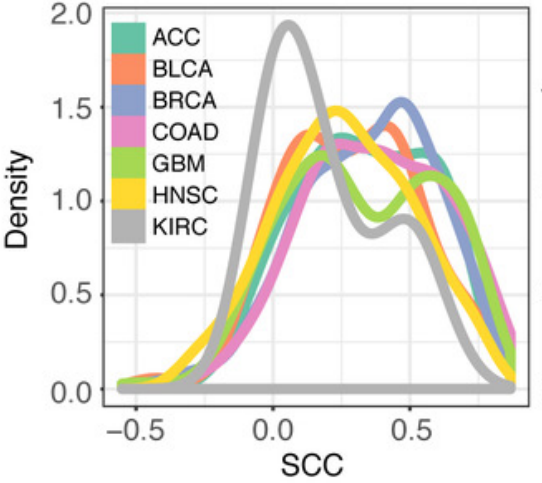

G)

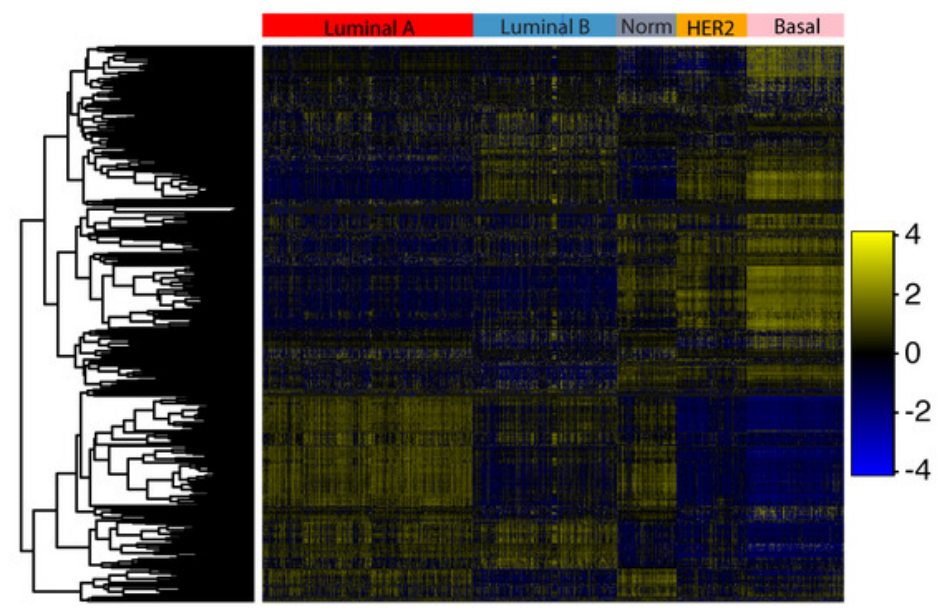

E)

F)
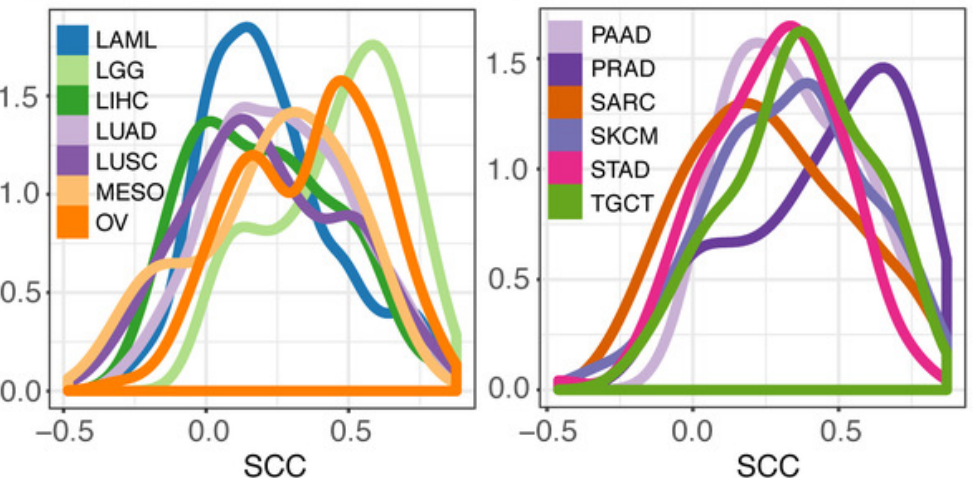


\section{Figure 4}

Systematic screening of prognostic IncRNAs in PRECOG compendium.

(A) Table showing the number of IncRNAs identified to be associated with patient prognosis across PRECOG cancer types using standard and robust meta-analysis methods. Top and bottom rows display different cutoffs for the Benjamini meta-FDR and Robust Benjamini-FDR, respectively. Haz. indicates hazardous and Pro. indicates protective. (B) Selected prognostic IncRNAs (Adjusted $p<0.001$ ) and their association with prognosis in each cancer type. White cells indicate associations with $p>0.05$ or IncRNAs whose expression cannot be inferred in that cancer type. Bar plots showing odds ratio indicating enrichment overlap between essential IncRNAs in (C) K562 cells and (D) IncRNAs associated with prognosis in hematopoietic cancers in PRECOG. Bar plots showing odds ratio indicating enrichment overlap between essential IncRNAs in (E) MDA-MB-231 cells and (F) IncRNAs associated with prognosis in breast cancer in PRECOG. Haz. indicates hazardous and Pro. indicates protective. Kaplan-Meier plots showing association of (G) TINCR, (H) H19, (I) EGOT, and (J) RP11-108P20.4 inferred expression with patient prognosis in selected brain, liver, breast cancer, and prostate cancer datasets from PRECOG. 
A)

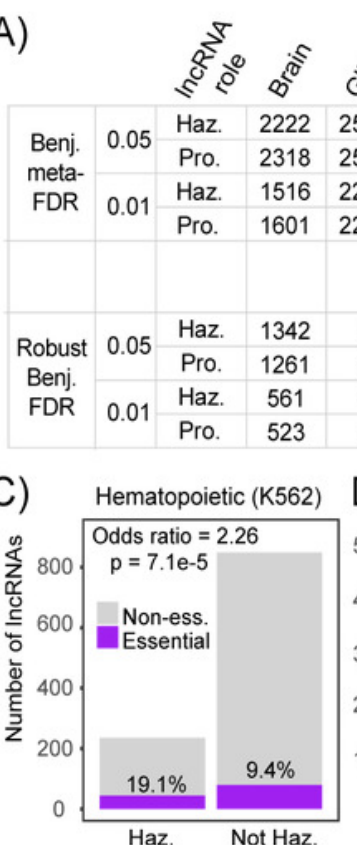

G) TINCR (Oberthuer, Brain)

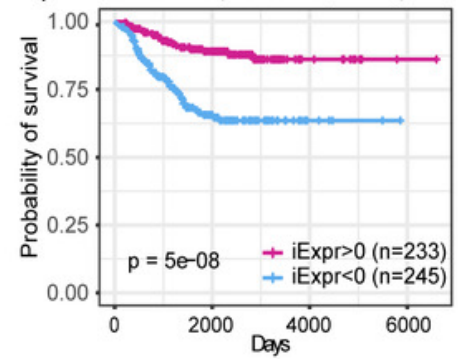

D) Hematopoietic (K562)

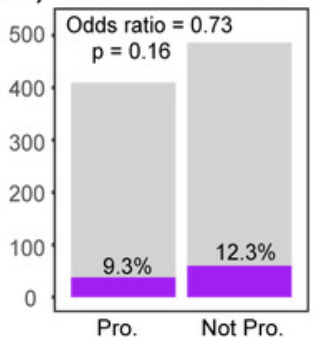

E) Breast (MDA-MB-231)

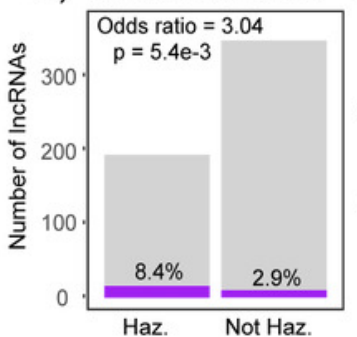

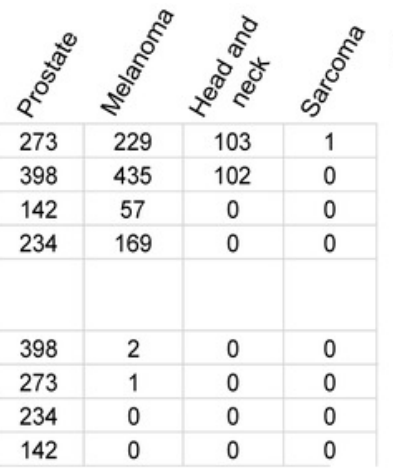

F) Breast (MDA-MB-231)

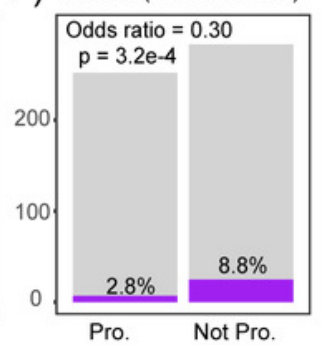

B)

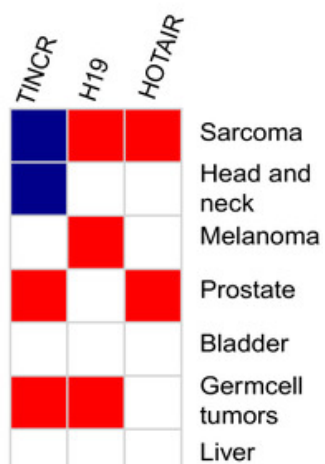

Liver

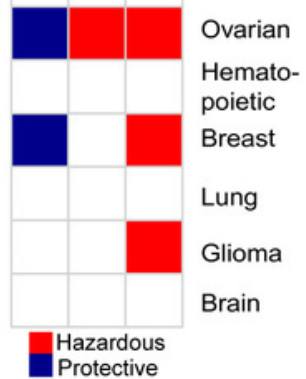

$\mathrm{H})$

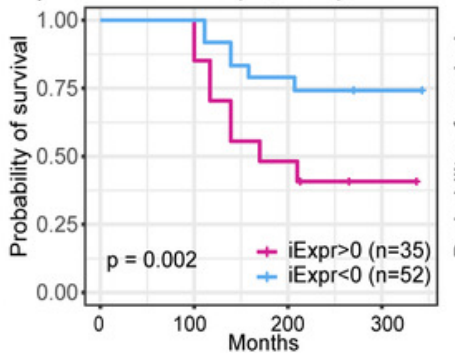

J) RP11-108P20.4 (Nakagawa, Prostate)

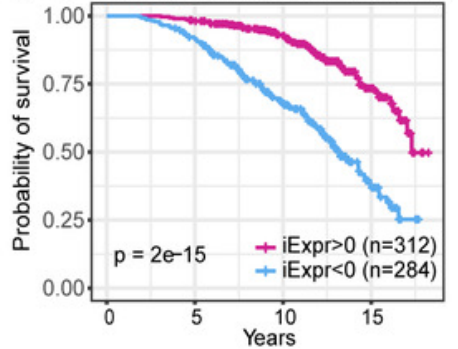




\section{Figure 5}

Prognostic IncRNAs identified in TCGA and PRECOG.

(A) Barplots showing odds ratios from enrichment analysis of prognostic IncRNAs identified in TCGA and PRECOG for LUAD, LGG, BLCA, LIHC, and LAML. Enrichment analysis was performed separately for IncRNAs with hazard ratios $>1$ (Red) and $<1$ (Blue). Vertical black line denotes an odds ratio of 1 . Scatterplots showing correlation of z-scores and meta zscores for all IncRNAs screened in TCGA and PRECOG, respectively, in (B) lung cancer, (C) brain cancer and (D) bladder cancer. Labeled points denote IncRNAs that have been characterized in previous literature. 
A)

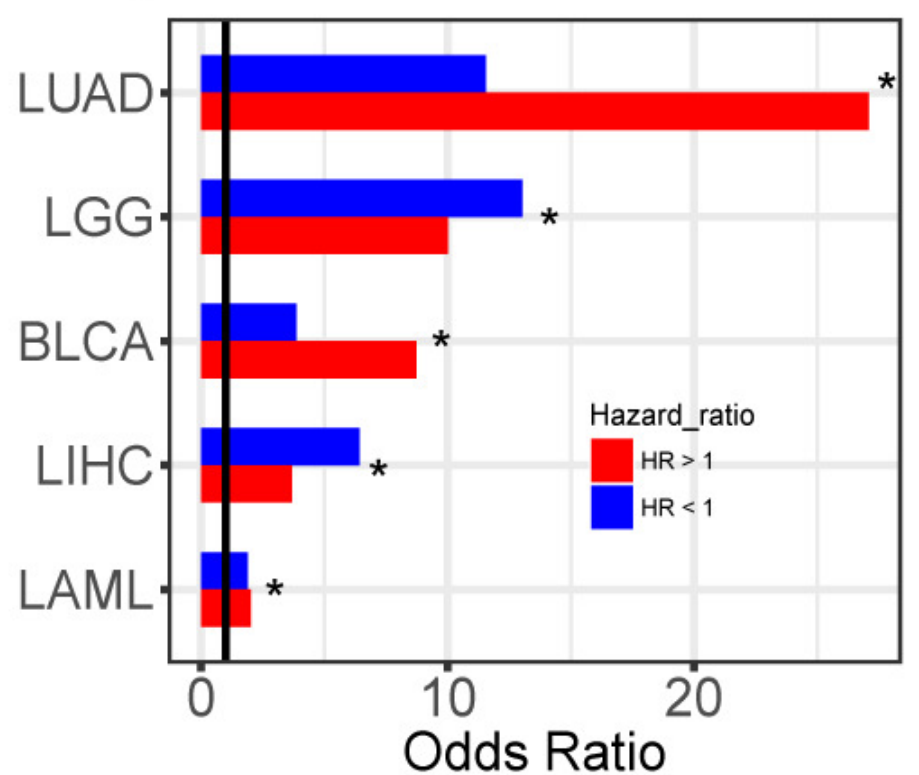

C) Braincancer (LGG)

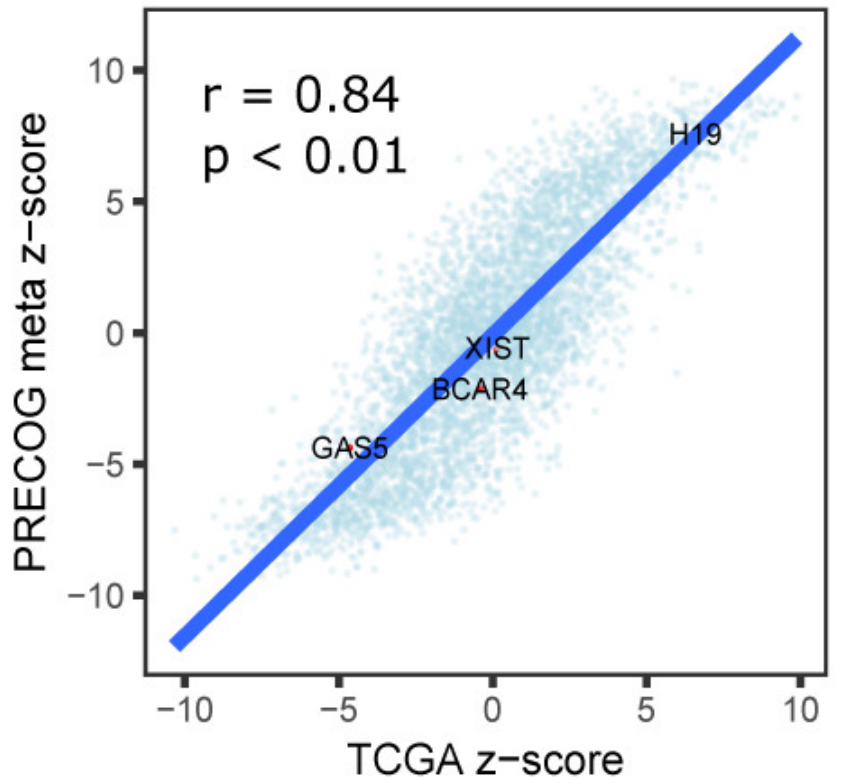

B) Lungcancer (LUAD)

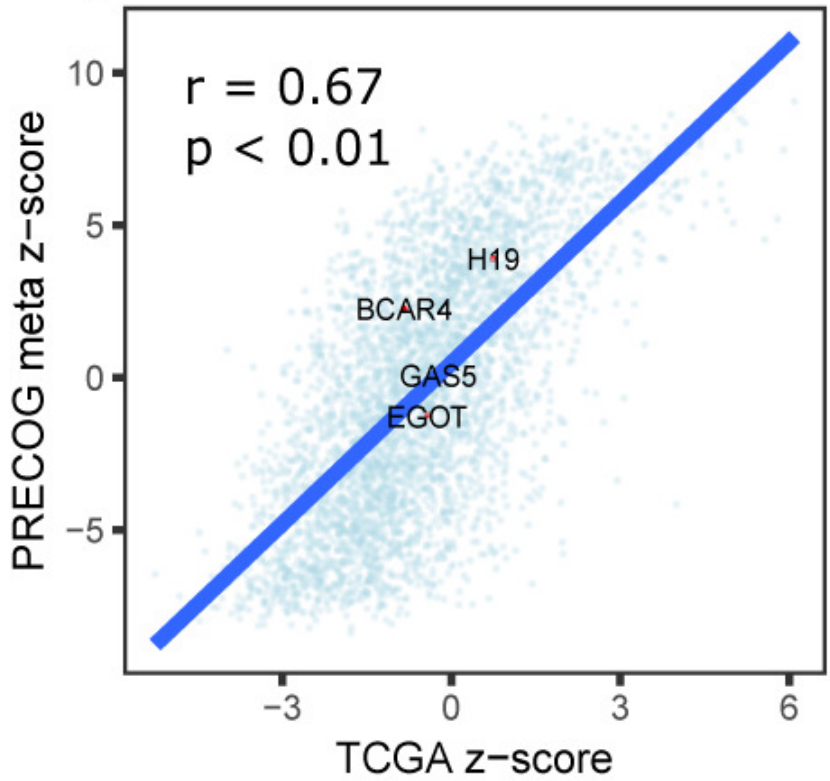

D) Bladdercancer (BLCA)

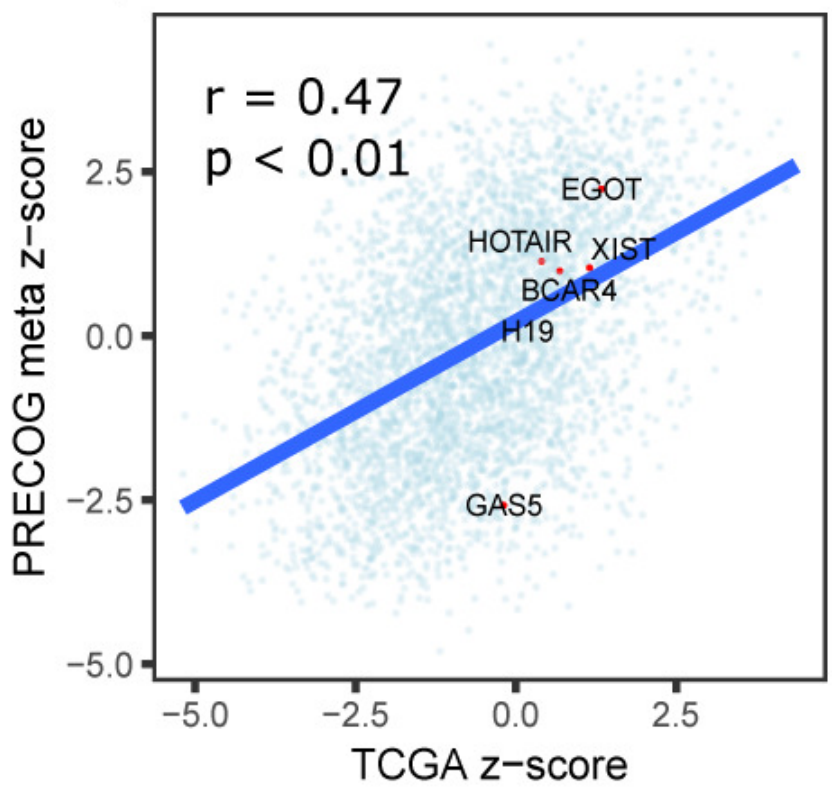




\section{Figure 6}

Enrichment of prognostic IncRNAs in genomic regions with copy number alterations.

Waterfall plots showing enrichment of (A) hazardous and (B) protective IncRNAs in amplified and deleted regions of the genome for each patient, respectively. $\log _{2}$ odds ratio $>0$ indicates enrichment and $\log _{2}$ odds ratio $<0$ indicates depletion. Purple bars indicate statistically significant enrichment. (C) High JRK inferred expression is associated with poor prognosis. (D) High JRK inferred expression is concentrated in regions with higher copy number signal across all METABRIC patients. (E) High CADM3-AS1 inferred expression is associated with favorable prognosis. (F) High CADM3-AS1 inferred expression is concentrated in genomic regions with lower copy number signal across all METABRIC patients. 


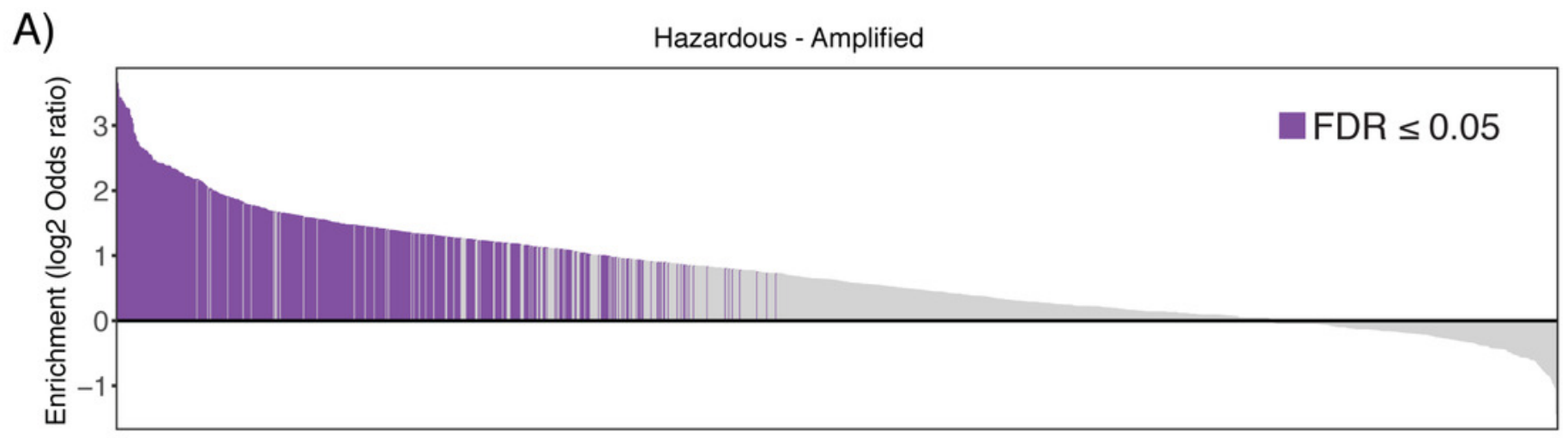

Patients

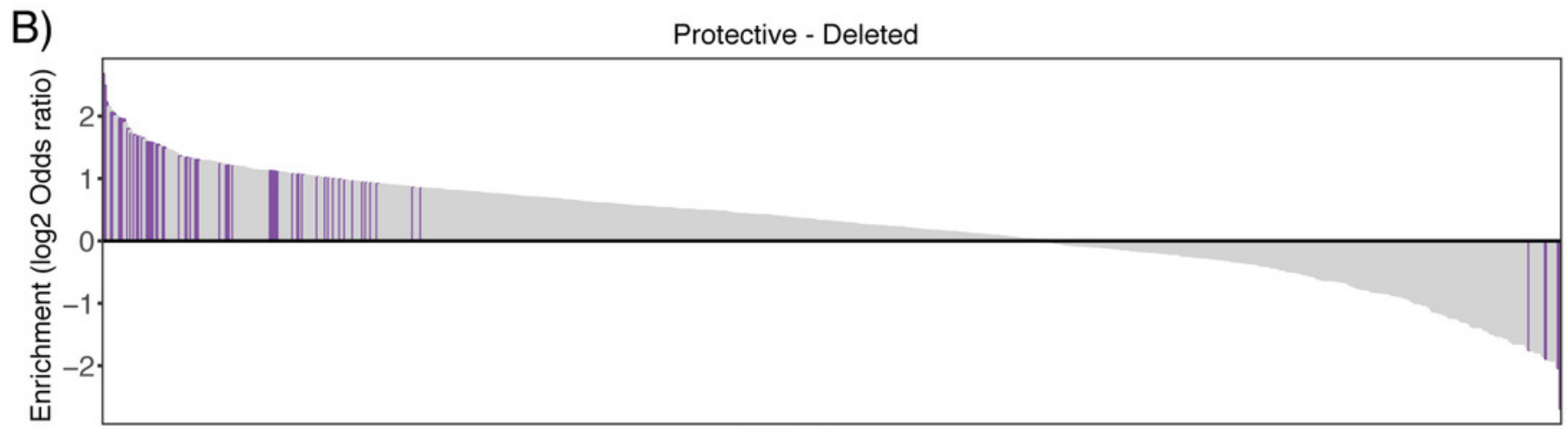

Patients

C)

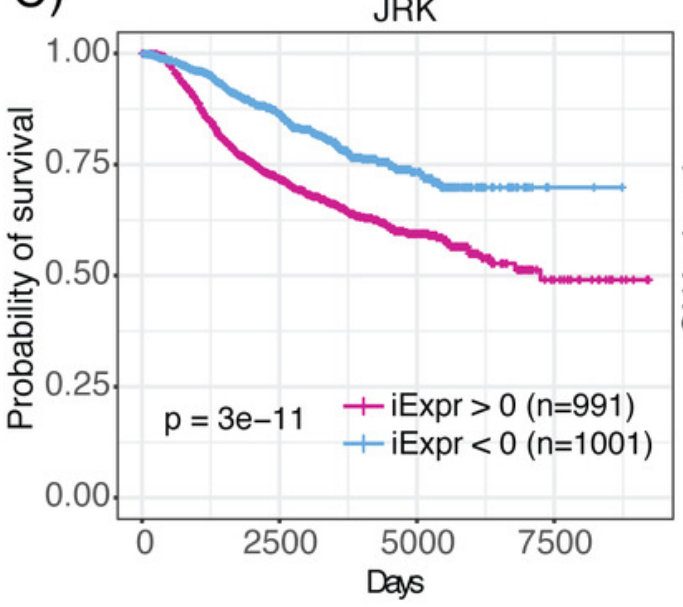

D)

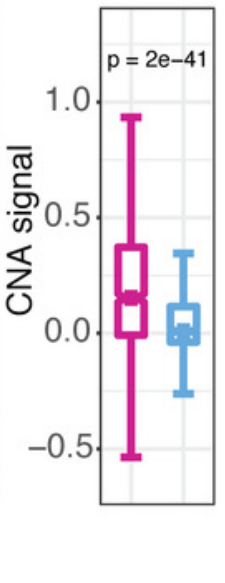

E)

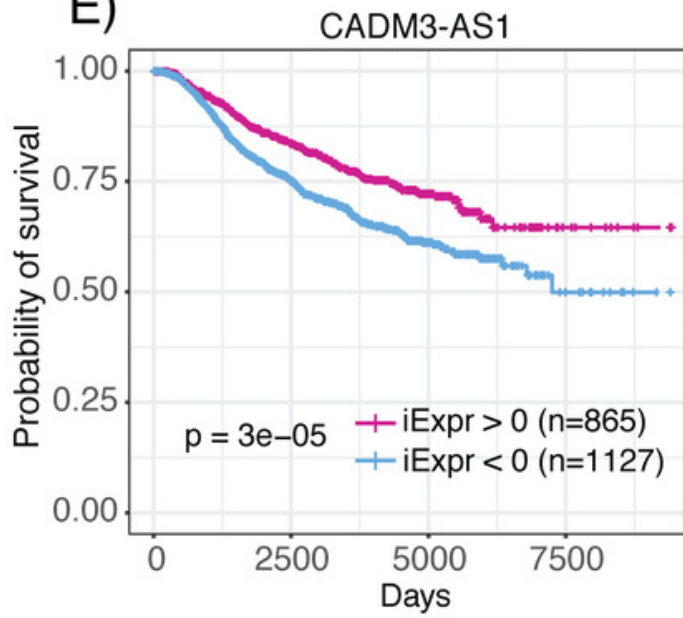

F)

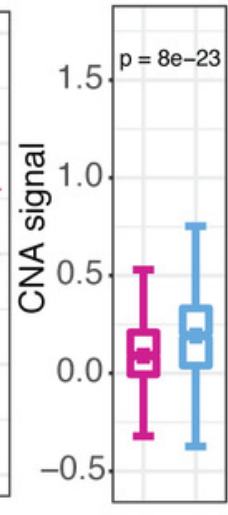

